\title{
Electron-Cyclotron-Current-Drive Efficiency in DEMO Plasmas
}

\author{
E. Poli, G. Tardini, H. Zohm, E. Fable \\ Max Planck Institute for Plasma Physics, \\ EURATOM Association, Garching bei München, Germany \\ D. Farina, L. Figini \\ Istituto di Fisica del Plasma CNR, \\ EURATOM-ENEA-CNR Association, Milano, Italy \\ N. B. Marushchenko \\ Max Planck Institute for Plasma Physics, \\ EURATOM Association, Greifswald, Germany \\ L. Porte \\ Centre de Recherches en Physique des Plasmas, \\ CRPP-EPFL, Lausanne, Switzerland
}

\begin{abstract}
The achievable efficiency for external current drive through electron-cyclotron waves in a demonstration tokamak reactor is investigated. Two possible reactor designs, one for steady state and one for pulsed operation, are considered. Beam propagation, absorption and current drive are modelled employing the beam tracing technique and including momentum conservation in electron-electron collisions. It is found that for midplane injection the achievable current drive efficiency is limited by second-harmonic absorption at levels consistent with previous studies. Higher efficiencies can be achieved by injecting the beams from the top of the machine, exploiting wave absorption by more energetic (less collisional) electrons. Current drive efficiencies competitive with those usually obtained by neutral beam current drive are reported. These optimum efficiencies are found for frequencies around $230 \mathrm{GHz}$ and $290 \mathrm{GHz}$ for the steady-state and the pulsed DEMO, supposed to operate at a magnetic field $B=5.84 \mathrm{~T}$ and $B=7.45 \mathrm{~T}$, respectively.
\end{abstract}




\section{INTRODUCTION}

To be commercially attractive, a fusion reactor based on the tokamak principle should be operated under stationary conditions, or at least over several hours with a short downtime in between (during which the central solenoid is recharged while the plant is still producing electricity). To this aim, the fraction of the plasma current induced by the transformer during the flattop should be zero or close to zero. This implies a maximization of the fraction of non-inductive current, which relies on one side on the bootstrap effect [1,2] and on the other side on externally driven currents, produced by the injection of electromagnetic waves or energetic neutral particles [3]. One of the schemes for external current drive commonly employed in magnetic-confinement fusion devices relies on the injection of mmwave beams that resonantly interact with the cyclotron motion of the electrons [4-7]. From the technological point of view, electron cyclotron current drive (ECCD) is considered the most mature among the heating and CD systems envisaged for ITER, due to the availability of reliable high-power sources and to the simplicity of the wave-plasma coupling. In spite of this, due to the lower CD efficiency (current driven per unit injected power) as compared with other CD methods, in particular neutral beam injection, ECCD alone is not considered as an option for ITER steady-state scenarios within the present design [8]. It should be considered, however, that the design parameters of present ECRH systems (including ITER) have not been selected with the main goal of maximizing the amount of driven current. Moreover, the attractiveness of a CD system in a power plant is determined, in addition to the CD efficiency, also by its efficiency in converting electrical power into injected power (wall-plug efficiency). To quote an example, conversion efficiencies up to $57 \%$ have been recently reported for ITER-relevant gyrotrons [9], which is higher than presently achieved for NBI systems. Finally, ECCD has the advantage that the launchers require just a small slot in the blanket and the first tritium barrier can be incorporated into the vacuum vessel.

The goal of the present paper is to assess the CD efficiency that can be reached with EC waves in a tokamak under reactor conditions. For this purpose, it is useful to introduce two figures of merit often employed in the investigations of CD efficiency. The first one is the dimensionless quantity $\zeta_{C D}$, defined as [10]

$$
\zeta_{C D}=\frac{e^{3}}{\varepsilon_{0}^{2}} \frac{n_{e} R_{0}}{T_{e}} \frac{I_{C D}}{P} \approx 32.7 \frac{n_{20} R_{\mathrm{m}} I_{\mathrm{A}}}{T_{\mathrm{keV}} P_{\mathrm{W}}},
$$

where $e$ is the elementary charge, $n_{e}$ is the electron density, $T_{e}$ is the electron temperature, 
$R_{0}$ is the major radius of the plasma, $I_{C D}$ is the total driven current, $P$ is the injected power and the numerical constant in the second step follows from expressing the various quantities in the units shown as a subscript (the density is in units of $10^{20} \mathrm{~m}^{-3}$ ). A second expression for the CD efficiency often used in the literature is

$$
\gamma_{C D}=\frac{n_{20} R_{\mathrm{m}} I_{\mathrm{A}}}{P_{\mathrm{W}}}
$$

sometimes denoted as $\eta_{20}$, which is dimensional and does not contain the temperature in the denominator. The dimensionless efficiency $\zeta_{C D}$ should capture the basic dependence of the CD efficiency on temperature due to the change of collisionality and therefore describe the effect on $\mathrm{CD}$ of varying the region in velocity space where the wave-particle interaction takes place, whereas $\gamma_{C D}$ is more useful as a measure of the effect of temperature variation on the total driven current, whose maximization is ultimately the goal of a CD system in a reactor. Peak on-axis ECCD estimated for ITER Scenario $2[11]$ results in $\zeta_{C D} \simeq 0.25-0.3$. An efficiency comparable to that obtained by NBCD implies $\zeta_{C D} \simeq 0.5-0.55$. In terms of $\gamma_{C D}$, typical numbers quoted for ITER are around 0.2 for ECCD and 0.3 for NBCD [8]; for DEMO, Ref. [12] quotes $\gamma_{C D}=0.2$ for ECCD at $200 \mathrm{GHz}$ and $\gamma_{C D}=0.45-$ 0.55 for NBCD depending on the acceleration voltage. As stated above, the design of the $170 \mathrm{GHz}$ ECRH/ECCD system for ITER does not imply an optimal CD efficiency, but is rather the result of a compromise between the state of the art in the technology and needs of different physics applications (heating, bulk CD, stabilization of MHD modes). In particular, it is known that higher wave frequencies would lead to increased efficiencies $[13,14]$. In the present study, no constraint is imposed on the choice of the parameters of the EC antenna (frequency, position, steering angles, etc.), since our goal is to evaluate the achievable efficiency for a set of consistent DEMO configurations and understand the relevant limiting factors. A study of the flexibility that should be allowed in the antenna setup in order to cover possible deviations of the plasma from the assumed scenario implies a knowledge of the design constraints which is still not available. It is noted, however, that a reactor will likely work around a very restricted operational point, so that experimental flexibility is not predicted to become a main design driver.

There is still no consensus on the global parameters of a demonstration power plant (cf. Table I in Ref. [15]). Two different options are considered in this paper. The first one (describing a tokamak operating under stationary conditions) is close to the ITER-98 
design, while the second one (corresponding to a pulsed machine) is assumed to have a higher magnetic field, larger major radius and larger aspect ratio [16, 17]. These configurations are described in Sec. II. The optimization of the ECCD efficiency is discussed in Sec. III. It is already stressed at this point that the CD modelling performed here, based on linear absorption and on the adjoint method [18] for the determination of the CD efficiency, includes the corrections for momentum conservation derived in Ref. [19, 20], that in general lead to a higher CD efficiency with respect to the case without momentum conservation (typically by about $20 \%$ for large injection angles and high current drive), in agreement with the results of quasi-linear calculations $[13,21]$. In order to allow a better understanding of the results of Sec. III, some considerations on ECCD under reactor conditions are developed in Sec. IV. A summary of the results and conclusions are presented in Sec. V.

\section{DEMO PARAMETERS}

The first DEMO configuration used in our ECCD calculations corresponds to a machine supposed to operate under steady-state conditions, with major radius $R_{0}=8.5 \mathrm{~m}$, minor radius $a=2.83 \mathrm{~m}$, on-axis magnetic field $B=5.84 \mathrm{~T}$, normalized pressure $\beta_{N}=2.95$ (a parameter list for both DEMO models described in this section is given in Table 1). Two sets of kinetic profiles, calculated using the transport code ASTRA [22], have been considered. The first one assumes a peaked density profile, according to recent theoretical studies predicting density peaking with decreasing collisionality [23-25], while the second set retains the same $\beta_{N}$ value but assumes flat density inside the $\mathrm{H}$-mode pedestal as in the most conservative assumptions for the ELMy H-mode scenario for ITER [26].

\begin{tabular}{|l|r|r|r|r|r|r|}
\hline \hline DEMO model & $B[\mathrm{~T}]$ & $R[\mathrm{~m}]$ & $R / a$ & $I_{p}[\mathrm{MA}]$ & $n_{e 0}\left[10^{19} \mathrm{~m}^{-3}\right]$ & $T_{e 0}[\mathrm{keV}]$ \\
\hline Steady-state (peaked/flat $\left.n_{e}\right)$ & 5.84 & 8.5 & 3 & $19.1 / 22.8$ & $15.0 / 9.3$ & $53 / 64$ \\
Pulsed (peaked/flat $\left.n_{e}\right)$ & 7.45 & 9.6 & 4 & $14.4 / 17.3$ & $16.8 / 10.4$ & $57 / 54$ \\
\hline \hline
\end{tabular}

Table 1. Parameters for steady state and pulsed DEMO considered in this paper. The two values for plasma current, central electron density and central electron temperature refer to the peaked-density and flat-density options, respectively.

More specifically, in the "peaked-density" case, the shape of the density and temperature profiles is taken according to ASDEX Upgrade "improved H-mode" shots (also known as 
"hybrid" mode of operation $[27,28])$ and the actual density is linked to the plasma current $I_{p}$ by imposing the Greenwald fraction [29] at the edge $\left(n_{20} / n_{G W}=1\right.$ at $\rho_{p}=0.9$, with $n_{G W}=I_{p} / \pi a^{2}$, where $I_{p}$ is expressed in MA and $a$ in $\mathrm{m}$, and $\rho_{p}$ is the square root of the normalized poloidal flux). The current itself is chosen to be the minimum current required for ignition, $P_{\text {loss }}=P_{\text {fus }} / 5$ (an H-mode enhancement factor $H=1.2$ is assumed), which turns out to be 19.1 MA for the peaked-density case and 22.8 MA for the flat-density case. This last value agrees with $I_{p}=23 \mathrm{MA}$ of Ref. [16], where also a flat density profile was assumed. The different values of the plasma current in the peaked and flat-density case explain the different density at the pedestal top in both cases. The temperature profile is re-scaled such as to maintain the targeted value of $\beta_{N}$. A similar approach is explained in detail in Ref. [30]. Density and temperature profiles are shown in Fig. 1.
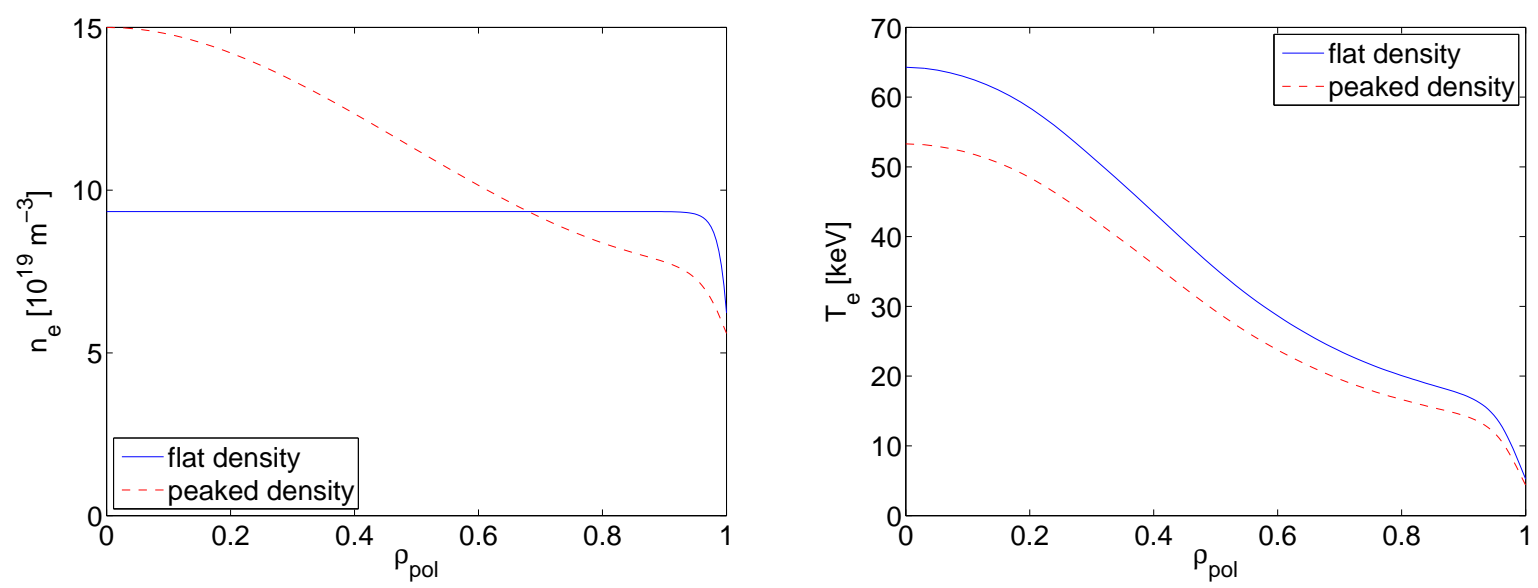

Fig. 1. Density and temperature profiles employed in ECCD calculations (steady-state DEMO) as a function of the square root of the normalized poloidal flux. Solid blue curves: "flat-density" case; dashed red curves: "peaked-density" case.

As mentioned in the Introduction, also machine parameters describing a pulsed device have been considered. To achieve a substantial pulse length (6-hours discharge in this case), a large central solenoid must be accommodated. Moreover, a large aspect ratio favours longpulse operation. Correspondingly, this design features $R_{0}=9.6 \mathrm{~m}, a=2.4 \mathrm{~m}, B=7.45$ $\mathrm{T}, \beta_{N}=2.6$. Density and temperature profiles have been calculated according to the same criteria as those for the steady-state design. Ignition is found for a plasma current of 14.4 and 17.3 MA for the peaked-density and flat-density case, respectively. 


\section{OPTIMIZATION OF THE ECCD EFFICIENCY}

The ECCD calculations reported in this paper are performed with the beam tracing code TORBEAM [31, 32], which employs the paraxial approximation to describe diffraction effects $[33,34]$. The modelling of absorption and current drive involves the fully-relativistic absorption routines written for the codes GRAY [35, 36] and TORAY [37], whereas the CD efficiency is computed using Lin-Liu's routine [38], augmented with the procedure to ensure momentum conservation in electron-electron collisions [19, 20, 39] first developed for the TRAVIS code. The effective charge is $Z_{\text {eff }}=2.57$ for the steady-state DEMO and $Z_{\text {eff }}=1.95$ for the pulsed DEMO. This high value of $Z_{\text {eff }}$ (which is detrimental for ECCD, as the driven current scales roughly as $\left.\left(5+Z_{\text {eff }}\right)^{-1}\right)$ has the purpose of ensuring a sufficient level of core radiated power needed to protect the divertor [16].
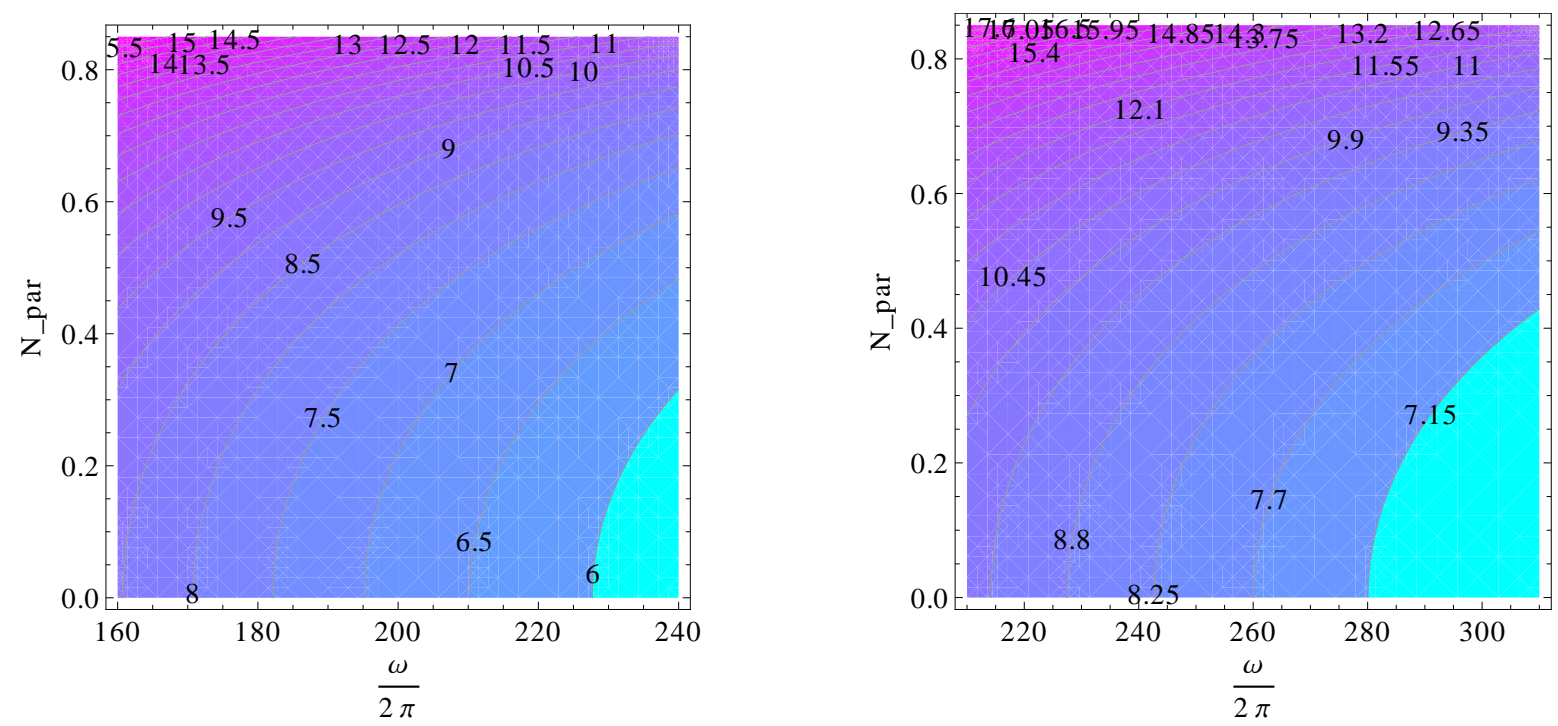

Fig. 2. Contour lines of major radii (in metres) at which the first-harmonic absorption starts to be possible, i. e. satisfying $\Omega(R) / \omega=\sqrt{1-N_{\|}^{2}}$ (left: steady-state DEMO parameters; right: pulsed DEMO parameters).

Before turning explicitly to the results of beam-tracing calculations, it is useful to recall that the (relativistic) resonance condition [40]

$$
\frac{n \Omega}{\omega}=\gamma-N_{\|} u_{\|}
$$

( $n$ is the harmonic number, $\Omega=e B / m_{e}$ the electron cyclotron frequency, $\omega$ the wave frequency, $\gamma=\sqrt{1+u^{2}}$ the relativistic Lorentz factor, where $u=p / m c$ is the normalized momentum, and $N_{\|}$the component of the refractive index vector $\mathbf{N}=c \mathbf{k} / \omega$ parallel to the equilibrium magnetic field) can be satisfied only for parallel momenta that lie between 
the two roots

$$
u_{\|, \mp}=\frac{N_{\|} n \Omega / \omega \mp \sqrt{(n \Omega / \omega)^{2}-\left(1-N_{\|}^{2}\right)}}{1-N_{\|}^{2}},
$$

so that first-harmonic absorption becomes possible starting from $R \leq \Omega_{0} R_{0} /\left(\omega \sqrt{1-N_{\|}^{2}}\right)$, where $\Omega_{0}$ is the cyclotron frequency at the geometric axis. This first resonance point for first-harmonic absorption is sometimes called the pinch point [13] and is characterized by

$$
u_{\|, p p}=\frac{N_{\|} \Omega}{\omega\left(1-N_{\|}^{2}\right)} .
$$

Fig. 2 reports the values of $R$ at which first harmonic absorption starts for different values of the wave frequency and of the parallel refractive index. Note that for high $N_{\|}$(optimum CD efficiency is usually found in a range $0.7 \lesssim N_{\|} \lesssim 0.8$ in the calculations presented in this paper) a small variation of $N_{\|}$results in a large shift of the first-harmonic resonance.
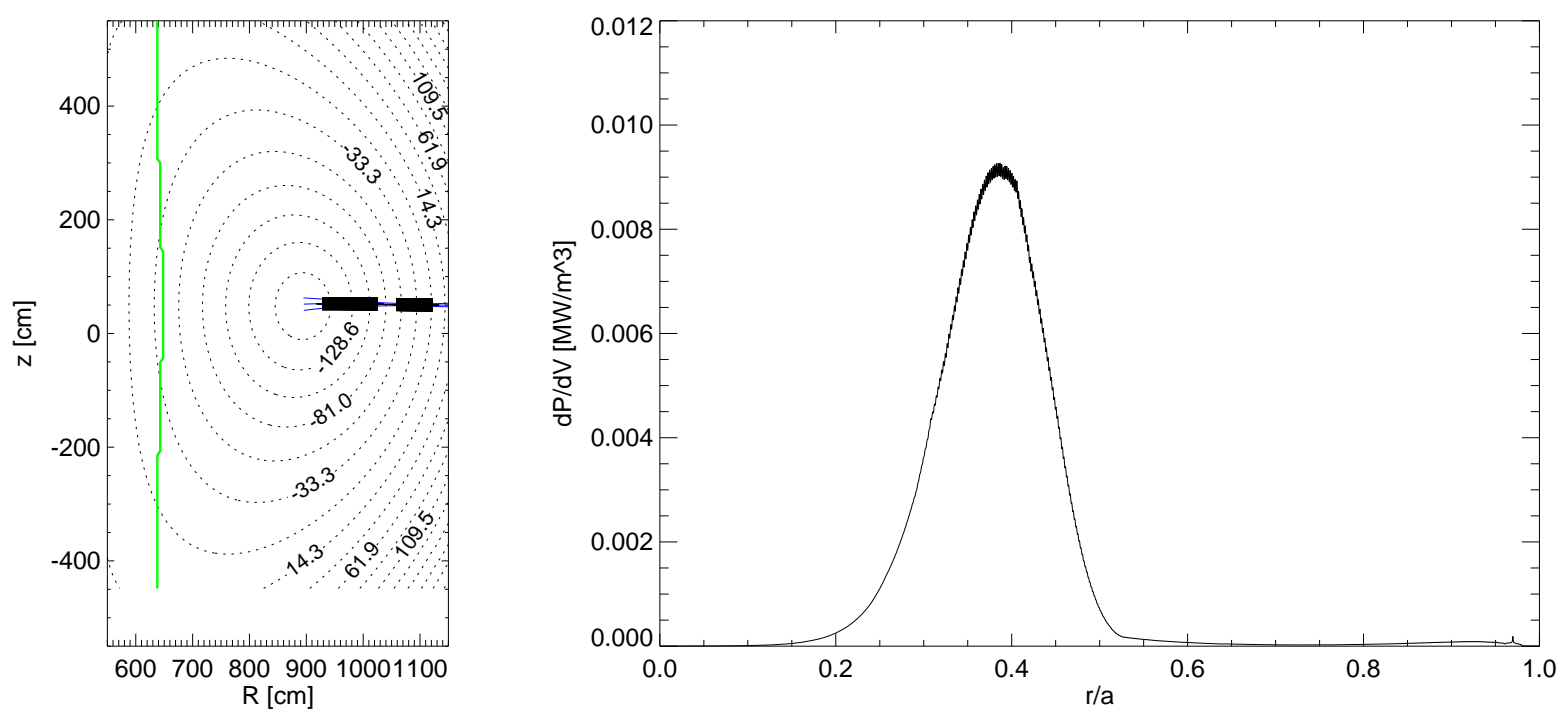

Fig. 3. Propagation of a Gaussian beam (left) and absorption profile (right) for horizontal injection in the plane of the magnetic axis (steady-state DEMO, peaked density; $\omega / 2 \pi=215$ GHz, toroidal injection angle $\left.\beta=40^{\circ}\right)$. The green nearly-vertical line in the left plot shows the position of the first-harmonic cold resonance $\Omega / \omega=1$. Slightly less than $10 \%$ of the power is absorbed by second cyclotron harmonic for these parameters.

Current drive for the steady-state DEMO design discussed in Sec. II is analyzed first and in more detail, as a high CD efficiency is particularly critical for stationary operation. We start with the performance of an equatorial launcher, injecting the EC power from the midplane at zero poloidal steering angle $\alpha$. Fig. 3 shows the propagation of a beam in the poloidal plane and the corresponding power deposition profile for a typical TORBEAM run. 

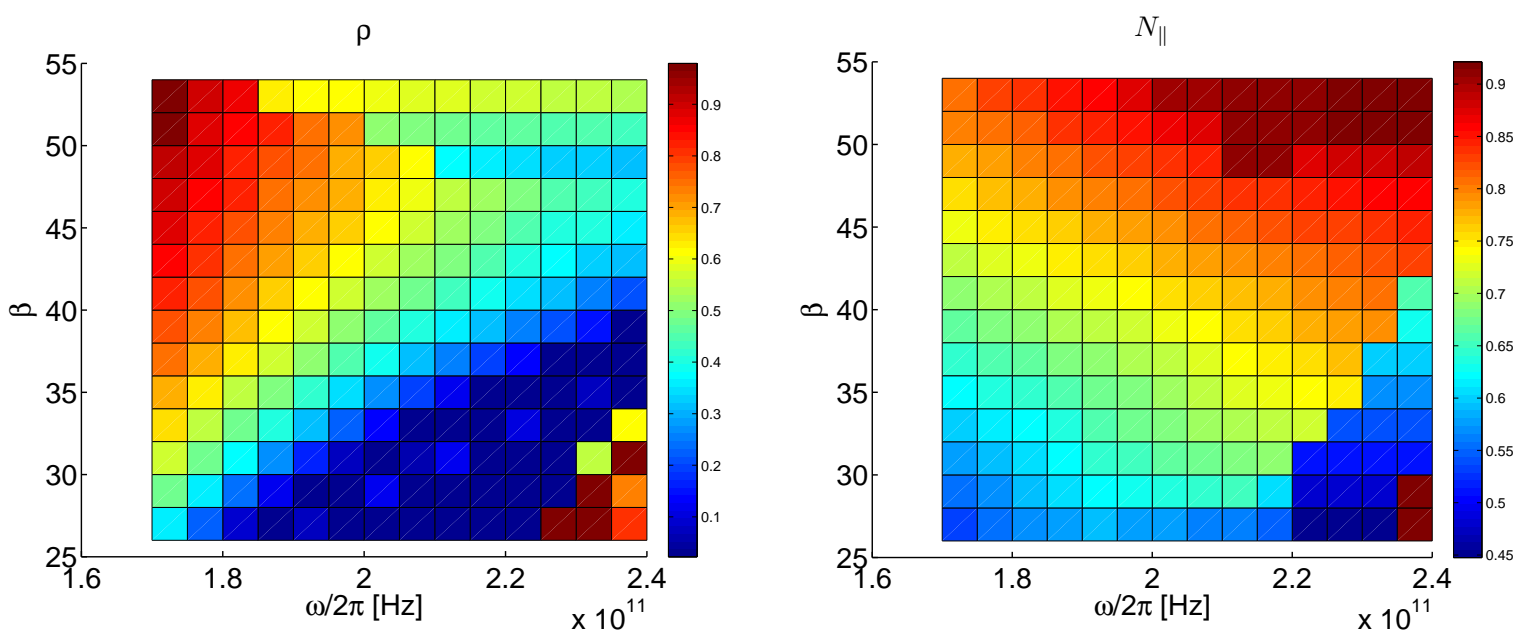

Fig. 4. Deposition radius (square root of the normalized poloidal flux) and parallel refractive index for propagation in the midplane (poloidal injection angle $\alpha=0)$ from $(R, Z)=(12.0,0.5)$ as a function of wave frequency and toroidal injection angle $\beta$ (steady-state DEMO, peaked density case).

In principle, maximum current drive on a given flux surface can be obtained on the high-field side, since this minimizes the impact of trapped particles. For this reason, it is beneficial to choose a high wave frequency, thus setting the resonance layer at smaller values of $R$. However, this implies that the wave travels through a broader region where no first-harmonic absorption is possible, but where second-harmonic absorption can be already significant. Since second-harmonic interaction reduces the power available for efficient (firstharmonic) current drive at the desired position, it has an undesired parasitic effect on the envisaged CD scheme [13, 41]. For DEMO parameters, it is found that parasitic absorption starts to be significant when the peak first-harmonic absorption is still on the low-field side of the magnetic axis, so that high-field-side CD turns out to be very challenging (down-shifted heating schemes relying on injection from the high-field side towards larger major radii turn out to be unsuitable for driving a large current, basically because of the unfavourable alignment between the beam and the magnetic field, which leads to low values of $N_{\|}$). Fig. 4 shows the normalized deposition radius $\rho_{p}$ (i. e. the radial position of maximum absorption) and the corresponding parallel refractive index $N_{\|}$for the peaked-density case as a function of the wave frequency and of the toroidal steering angle $\beta$. Increasing the wave frequency at constant $\beta$, the cold resonance is moved towards smaller values of $R$ and hence to smaller $\rho_{p}$ (the interaction takes place on the low-field side, as stated above). However, increasing simultaneously also $\beta$ (thus increasing the Doppler shift), the power can be delivered at a nearly constant radial position (Fig. 4, left panel), but with increasing 
$N_{\|}$(Fig. 4, right panel), with a beneficial effect on the CD efficiency. This can be seen in Fig. 5, where the current drive figures of merit $\zeta_{C D}$ and $\gamma_{C D}$, defined respectively in Eqs. (1) and (2), are shown.
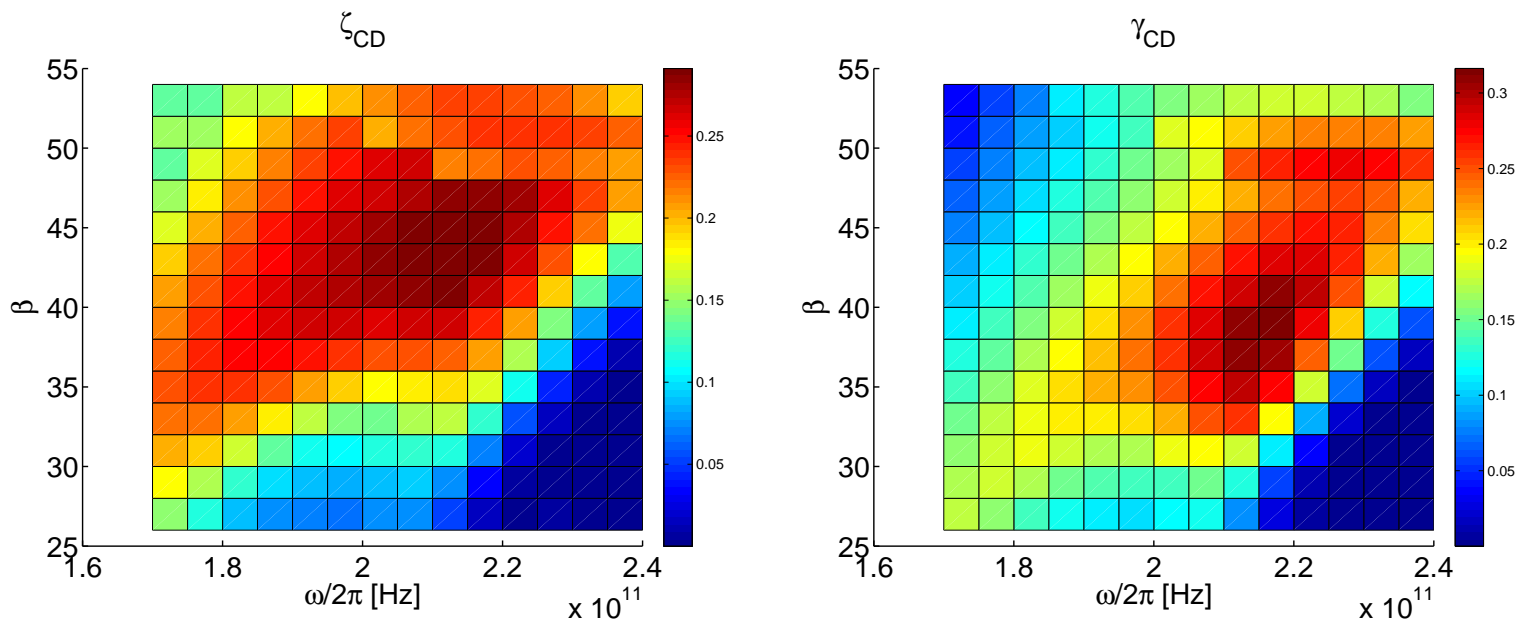

Fig. 5. Current drive efficiencies $\zeta_{C D}$ and $\gamma_{C D}$ for the same case as in Fig. 4.

Since the main goal of a CD system in a fusion reactor is to significantly contribute to the total plasma current, the quantity to be optimized is $\gamma_{C D}$ rather than $\zeta_{C D}$. Moreover, due to the fact that the scaling of the driven current with respect to $T_{e}$ is weaker than linear (cf. Sec. IV), optimizing $\zeta_{C D}$ would favour low temperatures, i. e. large values of $\rho_{p}$. This can be observed in Fig. 5, where the region of maximum $\zeta_{C D}$ shifts to larger $\rho_{p}$ with respect to the region of maximum $\gamma_{C D}$. It is stressed that in this paper the issue concerning the best radial location for ECCD in order to sustain a given scenario is not addressed; some remarks on this point are presented in Sec. V. The CD efficiency $\gamma_{C D}$ has a maximum around $\omega / 2 \pi=215 \mathrm{GHz}$ and $\beta=40^{\circ}$, with values just below 0.32 (the corresponding driven current being $I_{C D} \simeq 28 \mathrm{kA} / \mathrm{MW}$ ). Repeating the same exercise for the flat-density case, a maximum $\gamma_{C D}$ around 0.27 is found at nearly the same frequency and injection angle (note that, because of the lower density, the driven current in the flatdensity case is larger, $I_{C D} \simeq 33 \mathrm{kA} / \mathrm{MW}$ ). The slightly lower efficiency for the flat-density case as compared to the peaked-density case is due to the fact that the absorption takes place at larger $\rho_{p}$, where the fraction of trapped particles is higher (the flat-density case has smaller Shafranov shift, so that the resonance layer intersects the flux surfaces at larger $\left.\rho_{p}\right)$. It is noted that these values for frequency, injection angle and $\gamma_{C D}$ are in line with the results reported in Ref. [13], where a saturation (due to parasitic absorption) at around $\gamma_{C D}=0.3$ for temperatures above $30 \mathrm{keV}$ and for midplane injection was observed (as 
quoted earlier, the steady-state DEMO parameters considered here are in fact similar to the ITER parameters analyzed in Ref. [13]).
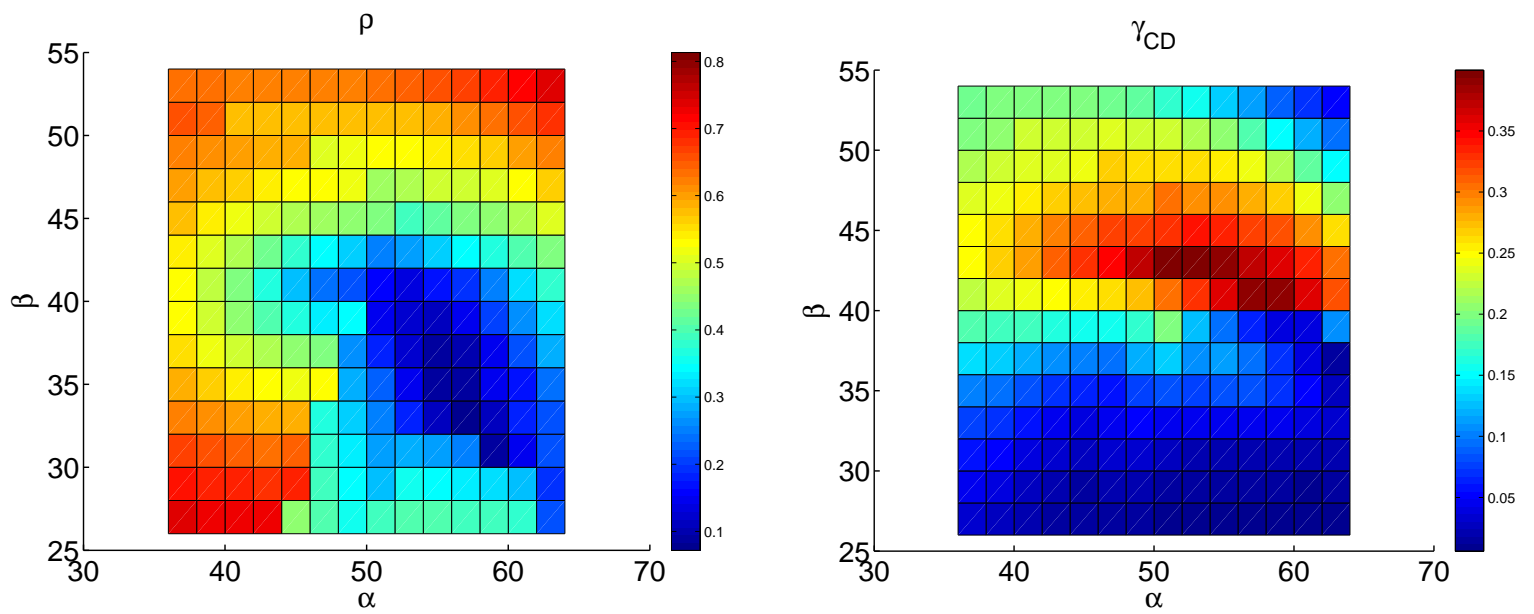

Fig. 6. Deposition radius and $C D$ efficiency for injection from the upper part of the vessel, $(R, Z)=$ $(10.5,3.5) \mathrm{m}$, as a function of the poloidal injection angle $\alpha$ and the toroidal injection angle $\beta$, for a wave frequency of $230 \mathrm{GHz}$ (steady-state DEMO, peaked density case).

The sensitivity of this scheme to second-harmonic absorption can be illustrated by increasing the frequency from 215 to $225 \mathrm{GHz}$, or decreasing the toroidal injection angle from $40^{\circ}$ to $35^{\circ}$, that leads to an increase of parasitic absorption from $6 \%$ of the total absorbed power to $31 \%$ and $23 \%$, respectively.

Clearly, the path through the region of parasitic absorption can be reduced by moving the EC antenna to smaller major radii. This can be achieved by placing it in the upper part of the machine, which also ensures good alignment for co-ECCD. The CD efficiency for a beam injected from $(R, Z)=(10.5,3.5) \mathrm{m}$ is shown in Fig. 6 . Values of $\gamma_{C D}$ close to 0.4 can be reached for $\omega / 2 \pi=230 \mathrm{GHz}$ with peak at $\rho_{p}=0.25$. This higher efficiency can be mainly ascribed to the fact that the beam energy is absorbed by more energetic (less collisional) electrons as compared to the case of midplane launch, according to the mechanism discussed later in Sec. IV. The angular range in the $(\alpha, \beta)$ plane for which these high CD efficiencies are obtained is relatively small (right panel of Fig. 6). As already observed with reference to Fig. 2, a small change in the injection geometry and hence in $N_{\|}$can result in a large shift of the first-harmonic resonance layer.

The dependence of the maximum CD efficiency and of the corresponding deposition radius on the wave frequency for different launch locations is plotted in Fig. 7. 

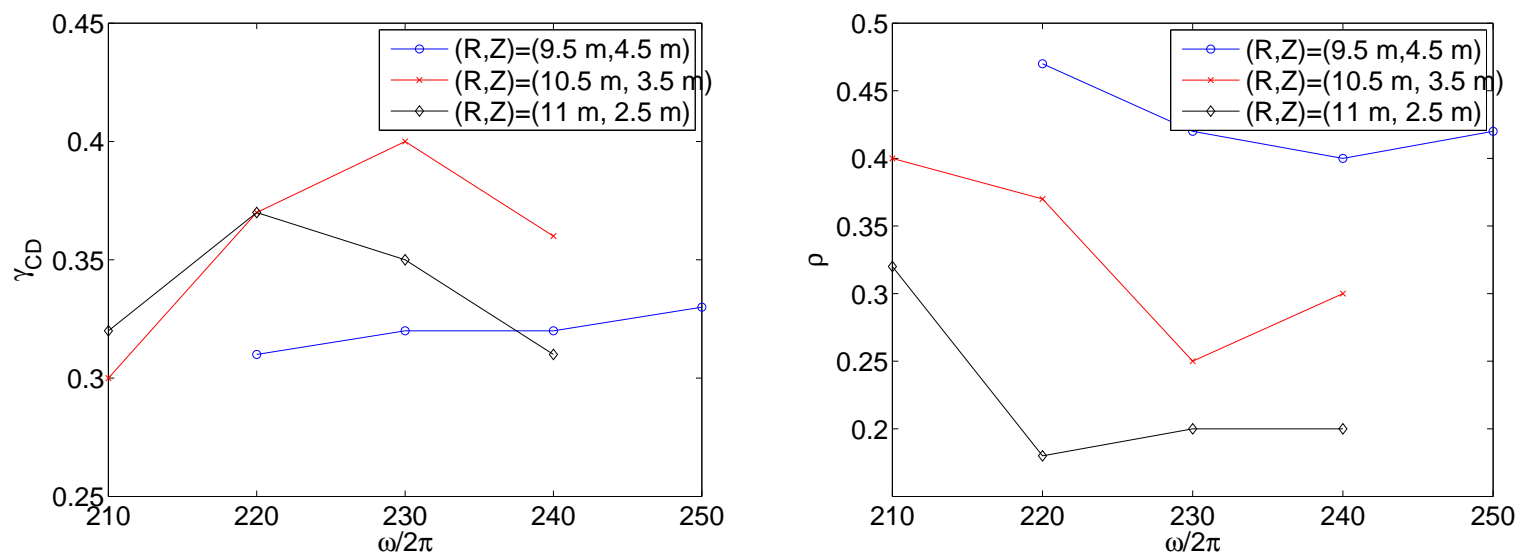

Fig. 7. Maximum CD efficiency (left) and corresponding radial location (right) as a function of the injected wave frequency for the launch positions indicated in the legend (peaked-density case).

It can be seen that by moving the antenna from the midplane to a more and more elevated position, the maximum efficiency is obtained for higher and higher frequencies. At the same time, the location of the deposition radius shifts outwards. The maximum efficiency is achieved where the effect of high $T_{e}$ on the CD efficiency is still not spoiled by the parasitic absorption. Efficiency values $\gamma_{C D}>0.3$ are obtained in a radial range $0.2 \lesssim \rho_{p} \lesssim 0.4$.

For top injection a similar trend as for equatorial injection is found when the peakeddensity case is replaced by the flat-density case, i. e. the CD efficiency decreases and the maximum efficiency moves towards larger values of $\rho_{p}$ (maximum $\gamma_{C D}=0.33$ at $\rho_{p}=$ $0.37)$.
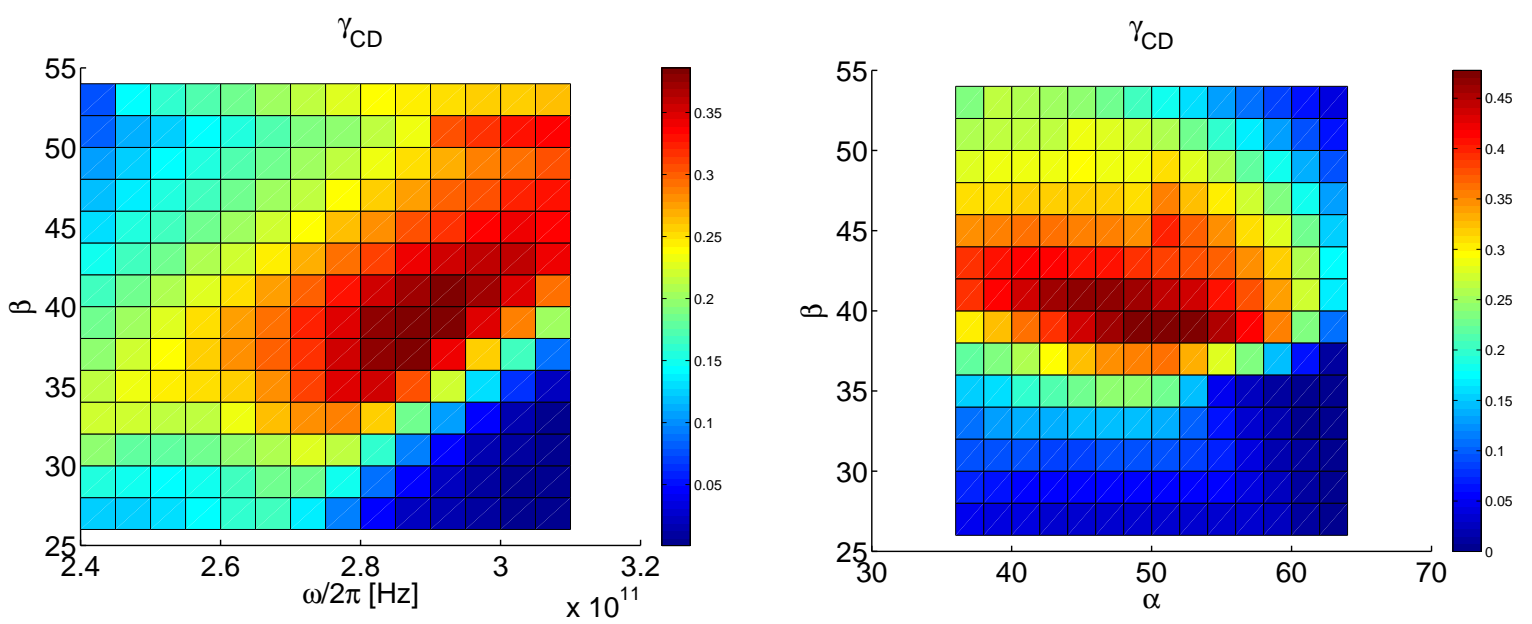

Fig. 8. CD efficiency for equatorial (left) and top injection (right) for the pulsed-DEMO design (peakeddensity case). Launch positions are $(R, Z)=(13.0,0.5) m$ and $(R, Z)=(12.0,3.0)$, respectively. The frequency in the top-injection case is $290 \mathrm{GHz}$

For the pulsed-DEMO design presented in Sec. II, similar qualitative trends as for the 
steady-state option are found. From a quantitative point of view, because of the much higher magnetic field presently foreseen for the pulsed DEMO in EU-studies, the optimum CD efficiency occurs at higher frequencies. A comparison between midplane and top injection is shown in Fig. 8. Again, injection from an upper port leads to higher CD efficiencies. The best efficiency is achieved for a frequency around $290 \mathrm{GHz}$. Because of the larger aspect ratio (smaller trapped-particle fraction) and the smaller value of $Z_{\text {eff }}$, the amount of driven current can be very high, resulting in $\mathrm{CD}$ efficiencies $\gamma_{C D}>0.45$.

Since these high values of $\gamma_{C D}$ are also due to the high temperatures foreseen in the DEMO options considered so far, a different design for the pulsed DEMO is shortly considered to conclude this section. The main global parameters are $R_{0}=9 \mathrm{~m}, a=2.25 \mathrm{~m}$, $B=6.8 \mathrm{~T}, \beta_{N}=2.25$. The central electron temperature $T_{e 0}$ is $19 \mathrm{keV}$ for the peakeddensity case $\left(n_{e 0}=16.1 \times 10^{19} \mathrm{~m}^{-3}\right)$ and $26 \mathrm{keV}$ for the flat-density case $\left(n_{e 0}=8.8 \times 10^{19}\right.$ $\left.\mathrm{m}^{-3}\right)$. For these temperatures, which are in the same range as those envisaged for the ITER ELMy H-Mode scenario, parasitic absorption is far less critical.
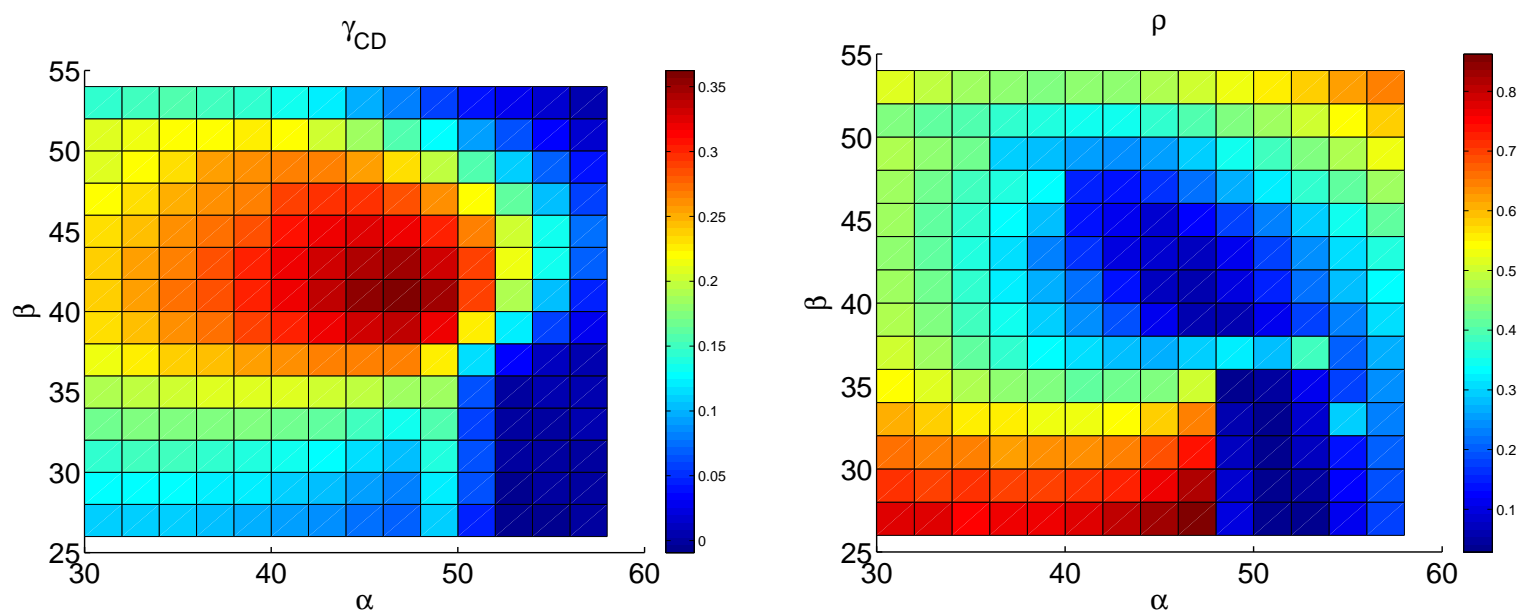

Fig. 9. Deposition radius and $C D$ efficiency for injection from the upper part of the vessel, $(R, Z)=(11,2.5)$ $m$, as a function of the poloidal injection angle $\alpha$ and the toroidal injection angle $\beta$, for a wave frequency of $270 \mathrm{GHz}$ (lower-temperature pulsed DEMO, peaked density).

The achievable CD efficiency and the corresponding deposition radius for a wave frequency of $270 \mathrm{GHz}$ and injection from an upper port located at $(R, Z)=(11,2.5) \mathrm{m}$ can be seen in Fig. 9. The maximum value of $\gamma_{C D}$ is around 0.36. At these temperatures, the centre of the plasma is not "screened" by parasitic absorption and the maximum CD efficiency occurs near the magnetic axis. 


\section{CURRENT DRIVE IN REACTOR-GRADE PLASMAS AND INTERPRETA- TION OF BEAM-TRACING RESULTS}

In order to achieve a better understanding of the results presented in Sec. III, some peculiarities of ECCD under very high temperatures, such as those envisaged for DEMO plasmas, should be kept in mind. Similar conditions, that have been considered in previous studies for the old ITER design [13], are approached only in simulations for ITER advanced scenarios [41]. In particular, two points are addressed in this section, namely the scaling of the CD efficiency with the electron temperature and the increased efficiency found for top injection.
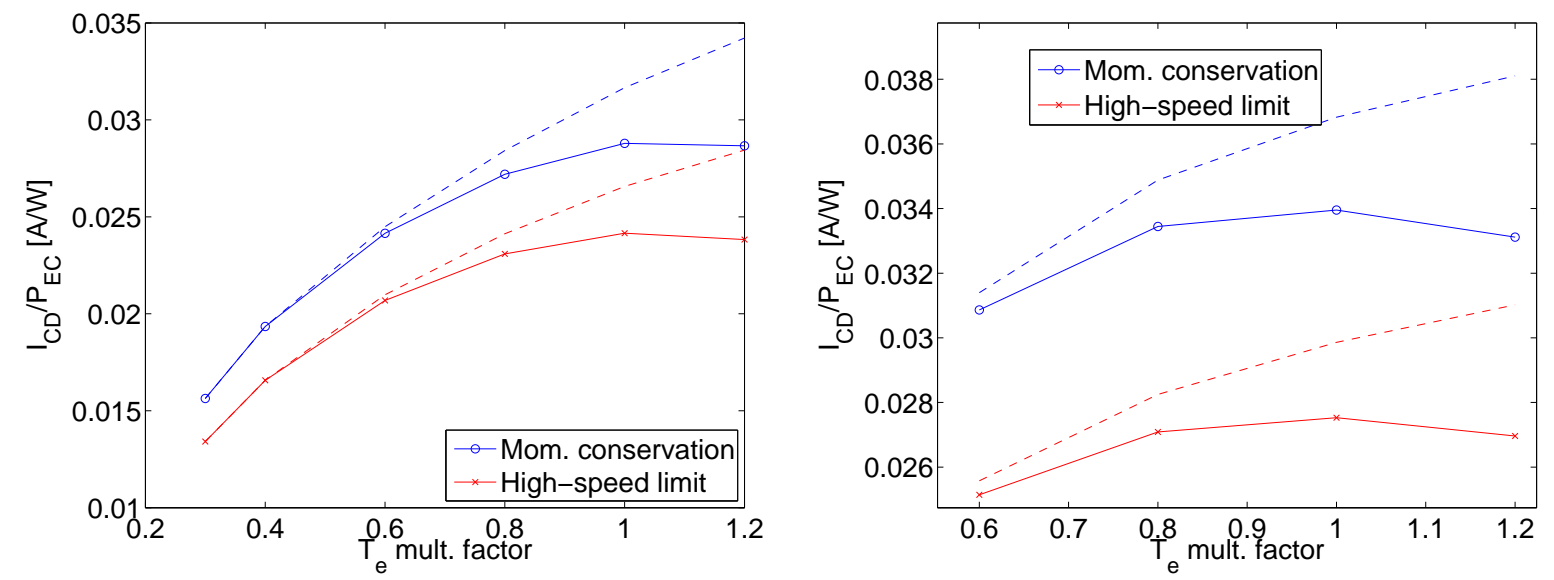

Fig. 10. Driven current (ampere per injected watt) for equatorial (left) and top injection (right), as discussed in Sec. III (steady-state DEMO, peaked-density case) obtained multiplying the nominal temperature profile by the factor shown on the horizontal axis (for lower temperatures than those reported in the plots, the absorption of the EC power starts to be incomplete). Circles refer to calculations that include momentum conservation, crosses to results obtained in the high-speed limit. The dashed curves are obtained dividing the driven current by the power fraction (decreasing with increasing temperature) available for first-harmonic absorption.

As already noted in the previous section, in the case of ECCD in very-high-temperature fusion plasmas, a major role is played by second-harmonic parasitic absorption, that depletes the beam energy available for first-harmonic current drive. In Fig. 10 it is shown that increasing the temperature while keeping all the other parameters unchanged (the beam trajectory, calculated from the cold-plasma dispersion relation, is not affected by a change in $T_{e}$ ), the driven current exhibits a sort of saturation at high $T_{e}$. In Fig. 10, the plasma parameters and injection locations are those found in Sec. III to correspond to optimum 
efficiency for the steady-state DEMO option, i. e. $\omega / 2 \pi=215 \mathrm{GHz}, \beta=40^{\circ}$ for midplane injection (left panel) and $\omega / 2 \pi=230 \mathrm{GHz}, \beta=42^{\circ}$ and $\alpha=50^{\circ}$ for top injection (right panel). The reason for this "saturation" of the driven current with increasing temperature is mainly the increasing second-harmonic absorption. The dashed lines in Fig. 10 show in fact that "renormalizing" the driven current to the energy effectively available at each temperature for first-harmonic current drive leads to a monotonous dependence on $T_{e}$. However, despite of this renormalization, the scaling of the CD efficiency with $T_{e}$ remains weaker than linear. The reason for this behaviour is discussed in the following (cf. Fig. 11). Incidentally, it is noted that Fig. 10 also confirms that the typical impact of including momentum conservation in ECCD calculation is of the order of $20 \%$, as mentioned in Sec. I.

To further clarify the dependence of the CD efficiency on the electron temperature, it is instructive to consider the evolution of the ratio between the driven current density $j$ and the absorbed power density $p$ while the beam propagates through the region of resonant interaction. In Fig. 11, $j / p$ is plotted as a function of the local temperature along the beam trajectory (only the first-harmonic region is considered). In both the left and right panels, the upper bunch of curves is obtained with momentum conservation, the lower bunch in the high-speed limit. In each bunch, the temperature profile is varied as in Fig. 10 multiplying the temperature profile by a factor between 0.6 and 1.2. The dashed lines connecting the symbols on the curves allow us to infer the dependence of the CD efficiency on the temperature, since equal symbols refer to equal positions along the ray.

At the start of first-harmonic absorption (i. e. at the pinch point where $\Omega / \omega=\sqrt{1-N_{\|}^{2}}$, marked by diamonds), the ratio $j / p$ is nearly independent from $T_{e}$ in the high-speed limit, while it slightly decreases with $T_{e}$ when momentum conservation is included. Later on, a nearly linear dependence of $j / p$ on the temperature can be observed at each position along the beam path. The slope of the corresponding straight line is not constant, but rather increases while the beam crosses the resonant region. At higher temperatures, when wave absorption is stronger, the current is driven closer to the pinch point (see also Fig. 12 that show the position of peak current drive $\max \left(\mathrm{d} I_{C D} / \mathrm{d} s\right)$, where $s$ is the arclength along the central ray). As we have just observed, closer to the pinch point the dependence of the CD efficiency on $T_{e}$ is weaker (in the limiting case of very strong absorption just after the pinch point, there would be no temperature dependence at all in the high-speed limit). This is the main reason for the less-than-linear scaling of the driven current with respect to $T_{e}$. 

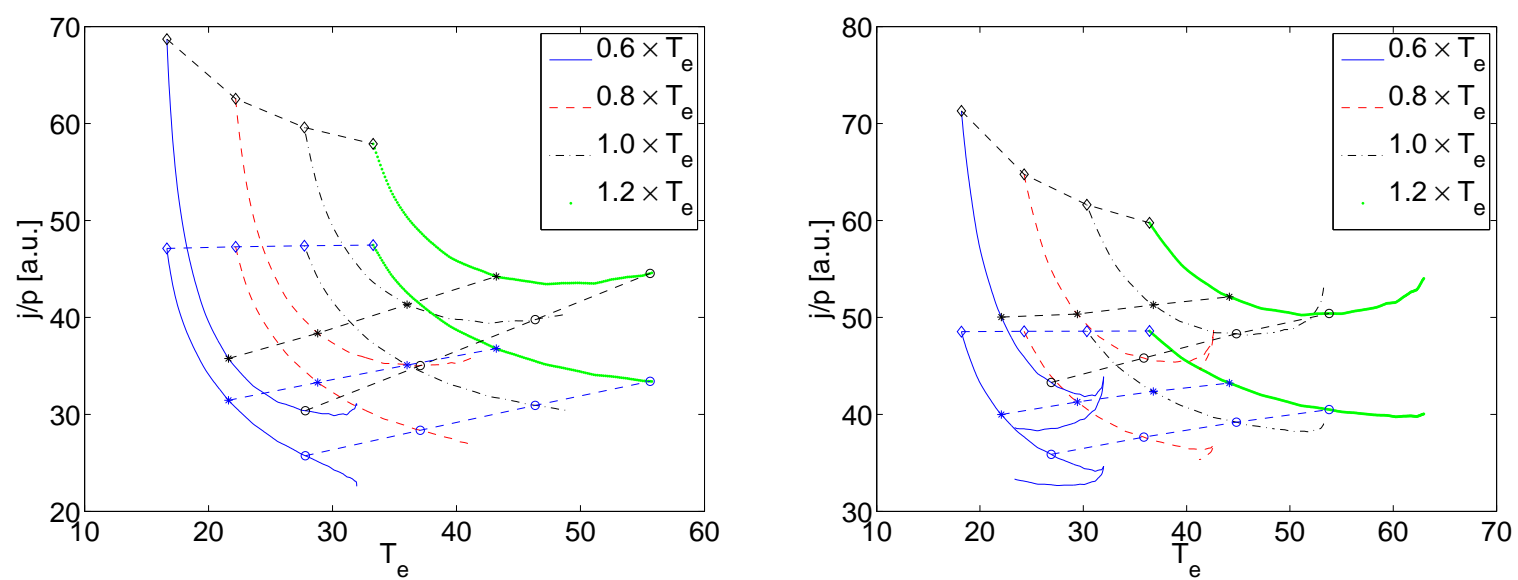

Fig. 11. Ratio $j / p$ as a function of the temperature along the ray for equatorial (left) and top injection (right). The nominal temperature profile (steady state DEMO, peaked density) is multiplied by 0.6 (blue solid curve), 0.8 (red dashed), 1.0 (black dash-dotted) and 1.2 (green dotted). The upper curves refer to momentum-conserving ECCD calculations, the lower curves to high-speed-limit results. Equal symbols along the curves correspond to equal positions along the ray.

This behaviour can be understood from the analytic expression for $j / p$, which can be written in the form [cf. e. g. Eq. (7) of Ref. [42]]

$$
\frac{j}{p} \propto \frac{\int_{u_{\|,-}}^{u_{\|,+}} \mathrm{d} u_{\|} \mathcal{P}\left(u_{\|}\right) \eta_{\mathbf{u}}\left(u_{\|}\right)}{\int_{u_{\|,-}}^{u_{\|,+}} \mathrm{d} u_{\|} \mathcal{P}\left(u_{\|}\right)}
$$

where $\mathcal{P}$ and $\eta_{\mathbf{u}}$ represent the normalized absorbed power and the specific current drive efficiency per unit momentum $\mathbf{u}$, respectively, and the resonance condition is used to express the dependences on $\mathbf{u}$ and $u_{\perp}$ in terms of $u_{\|}$. In Eq.(6), the dependence on temperature is mainly contained in the Maxwellian factor entering $\mathcal{P}$. Thus, at the first point in resonance, where the integration range collapses to the single point $u_{\|,-}=u_{\|,+}=u_{\|, p p}$, see Eq. (5), the factor $\mathcal{P}\left(u_{\|, p p}\right)$ simplifies from Eq. (6) and $j / p$ is proportional to $\eta_{\mathbf{u}}\left(u_{\|, p p}\right)$, which is independent on $T_{e}$ in the high-speed limit and decreases slightly with $T_{e}$ if momentum conservation is included, as shown in Fig. 10. As the resonance range in $u_{\|}$"opens", the factor $\eta_{\mathbf{u}}$, which increases with velocity (roughly as $u_{\|} u\left(u_{\|}\right)$), receives more and more weight as the temperature increases. The "local" linear scaling of $j / p$ with $T_{e}$ can be then inferred simply from a dimensional argument and is of the form $j / p \propto a+b T_{e}$ rather than $j / p \propto T_{e}$. The same scaling applies to the total driven current only if parasitic effects are absent and if width of the region where the wave-plasma interaction takes place does not change significantly with temperature. Both requirements are not met for high-CD schemes under reactor conditions. 

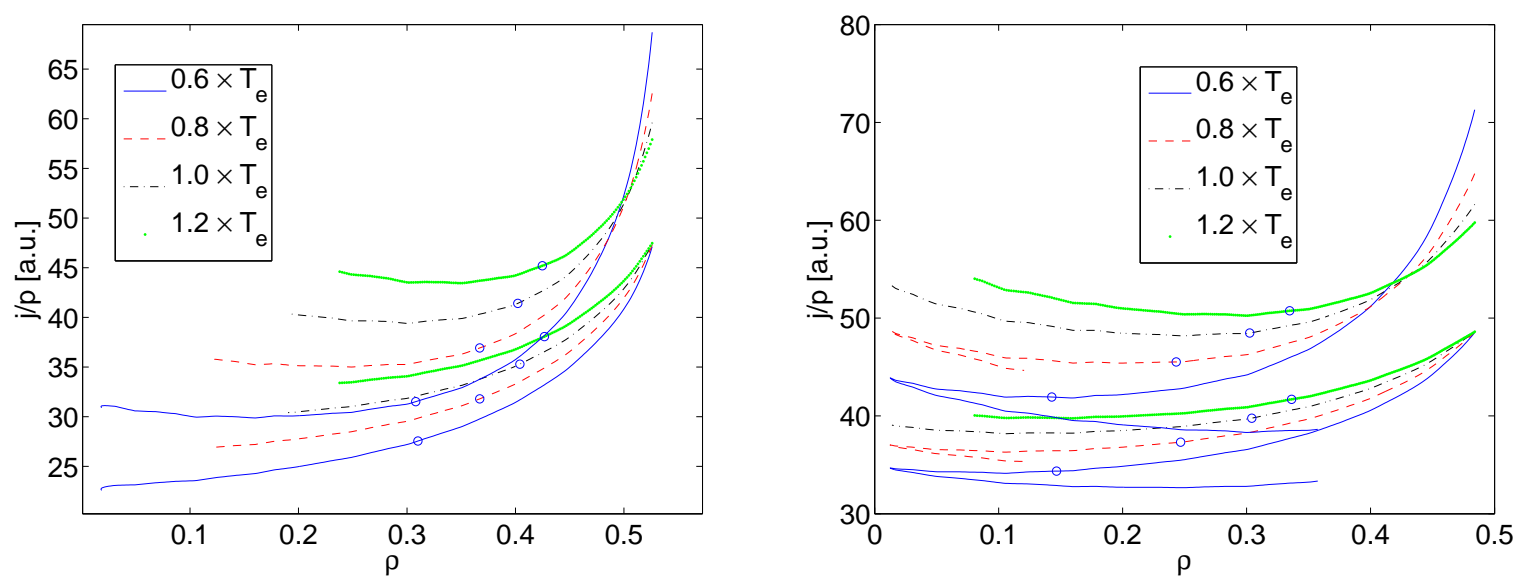

Fig. 12. Ratio $j / p$ as a function of the normalized radial coordinate $\rho_{p}$ calculated along the beam trajectory for equatorial (left) and top injection (right). As in Fig. 10 and 11, the nominal temperature profile (steady state DEMO, peaked density) is multiplied by 0.6 (blue solid curve), 0.8 (red dashed), 1.0 (black dashdotted) and 1.2 (green dotted). The upper curves refer to momentum-conserving ECCD calculations, the lower curves to high-speed-limit results. The open circles show the position of peak current drive $\mathrm{d} I / \mathrm{d} s$.

The previous discussion allows us also to understand an important feature of ECCD from a vertically displaced antenna as compared to midplane injection. In Fig. 12, the ratio $j / p$ of Fig. 11 is shown now as a function of $\rho_{p}$ along the ray. First harmonic absorption starts at $\rho_{p}$ slightly larger than 0.5 for equatorial launch and at $\rho_{p}$ slightly smaller than 0.5 for top launch, the difference being due to the higher wave frequency in the latter case. It is important to stress, however, that $N_{\|}$and $\Omega / \omega$ at the pinch point are very close in both cases. As seen in Fig. 12, $j / p$ decreases much faster with $\rho_{p}$ for midplane injection than for top injection. The reason for this behaviour is the fact that the variation of $\Omega(R) / \omega$ for oblique crossing of the resonance is slower than for horizontal crossing. Correspondingly, the resonant momentum range expressed by Eq.(4) opens more slowly. Since the integrands involved in $j / p$, cf. Eq. (6), peak near the lower integration bound $u_{\|,-}$because of the Maxwellian factor in $\mathcal{P}\left(u_{\|}\right)$, if $u_{\|,-}$decreases more slowly from its value at the pinch point, the wave is damped on faster electrons and the Fisch-Boozer effect [43] is enhanced. The values of $j / p$ at peak current drive are about $20 \%$ higher for top injection than for equatorial injection, accounting for most of the efficiency increase observed in TORBEAM calculations (the remaining gain in CD can be ascribed to trapped-particle effects, since for top injection the absorption is not located in the midplane as for equatorial injection). At the same time, the damping becomes weaker and the absorption profile broadens, as shown by the different $\rho_{p}$-ranges in the left and right panel of Fig. 12. The process just described 
can be considered as the relativistic, low-field-side counterpart of the effect discussed by Karney and Fisch [44] for the case of X-mode current drive from the high-field side.

\section{SUMMARY AND CONCLUSIONS}

Before summarizing the results of this paper, it is useful to add some remarks concerning the theoretical model adopted here to calculate propagation and absorption of EC wave beams. The use of the cold-plasma dispersion relation to determine the beam path should be well justified, since warm-plasma effects are reportedly largest for close-to-perpendicular propagation [45], whereas in this paper high $N_{\|}$-values are considered. However, an explicit check of the validity of this conclusion would be desirable. One could also question the validity of the paraxial approximation for schemes with significant oblique incidence onto the resonance layer. In this case, good confidence is given by recent results showing that the paraxial technique is in very good agreement with the exact solution of the wave equation obtained in a simplified planar geometry intended to mimic the conditions typical for heating and CD from the ITER upper launcher $[46,47]$. The combination of linear absorption and adjoint determination of the CD efficiency offers a well-benchmarked, reliable tool for the investigation of the CD efficiency. The broad absorption profiles typical of high-CD injection geometries result generally in relatively low power densities, ruling out quasi-linear effects [48] even for an injected power of several hundred MW (except possibly for the very centre of the plasma column, which is however usually not affected by the peak EC deposition in our simulations). An evaluation of synchrotron losses on the CD efficiency [49] is still to be addressed.

This paper shows that a comparatively high ECCD efficiency can be obtained in reactorgrade plasmas. The estimates for the figure $\gamma_{C D}$ for the steady-state DEMO design exhibit peak values between 0.3 and 0.4 depending on the assumptions on the kinetic profiles, and even above this value for the high-magnetic field, pulsed reactor considered here. The maximum efficiency is usually achieved at poloidal radii around $\rho_{p} \simeq 0.3$, where high temperatures are attained at still low parasitic absorption. The high efficiency reported here is due to several factors. First of all, the EC driven current has been computed including momentum conservation, which leads to higher values (by about 20\%) as compared to the the high-speed-limit scheme usually employed in linear calculations and in systems codes 
(momentum conservation was included in the quasi-linear simulations of Ref. [13], where indeed similar CD efficiencies for equatorial injection were reported). Moreover, the electron temperatures resulting from our assumptions on the density profile and on the target $\beta_{N}$ are somewhat higher than those considered in previous analyses. However, to circumvent the saturation of the CD efficiency at high temperatures due to increasing parasitic absorption, the injection position and the frequency must be selected carefully. High CD efficiency is obtained moving the launch position towards the high-field side. This can be achieved by injecting the beams from a port in the upper part of the vessel.

The optimum beam frequencies emerging from the DEMO parameters considered in this study are in a range around $230 \mathrm{GHz}$ for the steady-state and $290 \mathrm{GHz}$ for pulsed operation. A high CD efficiency is clearly more crucial for a stationary than for a pulsed tokamak, so that the constraints on the frequency could be relaxed in the latter case. Anyhow, this implies a serious challenge to the developers of gyrotron sources. On the other hand, one could observe that this applies also to other possible candidates for bulk current drive in a tokamak reactor, as e. g. neutral beam injection at 1 to $2 \mathrm{MeV}$.

The optimization of the CD efficiency is only a first step towards the design of a CD system for reactor operations. The optimum ECCD configuration depends on the kind of plasma scenario envisaged for (nearly) steady-state discharges. In general, it can be assumed that the "best" location for ECCD will result from the balance between the need for high CD efficiency (which would privilege the hotter plasma core) and that for high bootstrap fraction (implying that $q$ should not drop to too low values, which is of course also desirable for stability reasons). In this sense, one could speculate that the fact the peak CD efficiency is found here around $\rho_{p} \approx 0.3$ matches these requirements. Obviously, since this system is supposed to drive a significant part of the plasma current, the effects of the injection of, say, at least $200 \mathrm{MW}$ on a given magnetic configuration and on the corresponding profiles is to be investigated self-consistently, as done e. g. in Ref. [12]. This study is planned for the near future.

\section{ACKNOWLEDGMENTS}

We would like to dedicate this paper to the memory of Grigori V. Pereverzev.

This work has been partly performed within the frame of the EFDA Power Plant Physics 
\& Technology Work Programme under task WP12-DAS-HCD01-T05.

[1] R. J. Bickerton, J. W. Connor, and J. B. Taylor, Nature Physics 229, 110 (1971).

[2] A. G. Peeters, Plasma Physics and Controlled Fusion 42, B231 (2000).

[3] C. Gormezano et al., Progress in the ITER Physics Basis Chapter 6: Steady State Operation, Nuclear Fusion 47, S285 (2007).

[4] M. Bornatici, R. Cano, O. De Barbieri, and F. Engelmann, Nuclear Fusion 23, 1159 (1983).

[5] V. Erckmann and U. Gasparino, Plasma Physics and Controlled Fusion 36, 1869 (1994).

[6] T. C. Luce, IEEE Transactions on Plasma Science 30, 734 (2002).

[7] R. Prater, Physics of Plasmas 11, 2349 (2004).

[8] F. Wagner et al., Plasma Physics and Controlled Fusion 52, 124044 (2010).

[9] K. Sakamoto et al., Nuclear Fusion 49, 095019 (2009).

[10] T. C. Luce et al., Physical Review Letters 83, 4550 (1999).

[11] G. Ramponi, D. Farina, M. A. Henderson, E. Poli, G. Saibene, O. Sauter, H. Zohm, and C. Zucca, Nuclear Fusion 48, 054012 (2008).

[12] J. Garcia et al., Nuclear Fusion 48, 075007 (2008).

[13] R. W. Harvey et al., Nuclear Fusion 37, 69 (1997).

[14] ITER Physics Basis Expert Group on Energetic Particles, Heating and Current Drive, ITER Physics Basics Editors: Chapter 6: Plasma Auxiliary Heating and Current Drive, Nuclear Fusion 39, 2495 (1999).

[15] H. Zohm, Fusion Science and Technology 58, 613 (2010).

[16] D. J. Ward and W. E. Hen, EFDA Task Report TW6-TRP-002 (European Fusion Development Agreement, 2007).

[17] D. J. Ward, Plasma Physics and Controlled Fusion 52, 124033 (2010).

[18] T. M. Antonsen and K. R. Chu, Physics of Fluids 25, 1295 (1982).

[19] N. B. Marushchenko, H. Maassberg, and Y. Turkin, Nuclear Fusion 48, 054002 (2008).

[20] N. B. Marushchenko, H. Maassberg, and Y. Turkin, Nuclear Fusion 49, 129801 (2009).

[21] N. B. Marushchenko et al., Physics of Plasmas 18, 032501 (2011).

[22] G. V. Pereverzev and P. N. Yushmanov, ASTRA: Automated System for Transport Analysis in Tokamaks (IPP Report 5/98, 2002). 
[23] C. Angioni et al., Physical Review Letters 90, 205003 (2003).

[24] G. V. Pereverzev et al., Nuclear Fusion 45, 221 (2005).

[25] M. Greenwald et al., Nuclear Fusion 47, L26 (2007).

[26] T. Casper et al., Nuclear Fusion 52, accepted for publication (2012).

[27] A. Staebler et al., Nuclear Fusion 45, 617 (2005).

[28] A. C. C. Sips et al., Nuclear Fusion 47, 1485 (2007).

[29] M. Greenwald et al., Nuclear Fusion 28, 2199 (1988).

[30] G. Tardini et al., Nuclear Fusion 49, 075004 (2009).

[31] E. Poli, G. V. Pereverzev, A. G. Peeters, and M. Bornatici, Fusion Engineering and Design 53, 9 (2001).

[32] E. Poli, A. G. Peeters, and G. V. Pereverzev, Computer Physics Communications 136, 90 (2001).

[33] G. V. Pereverzev, Reviews of Plasma Physics 19, 1 (1996).

[34] G. V. Pereverzev, Physics of Plasmas 5, 3529 (1998).

[35] D. Farina, Fusion Science and Technology 52, 154 (2007).

[36] D. Farina, Fusion Science and Technology 53, 130 (2008).

[37] E. Westerhof, Implementation of TORAY at JET (Rijnhuizen Report RR-89-183, 1989).

[38] Y. R. Lin-Liu, V. S. Chan, and R. Prater, Physics of Plasmas 10, 4064 (2003).

[39] N. B. Marushchenko, C. D. Beidler, and H. Maassberg, Fusion Science and Technology 55, 180 (2009).

[40] M. Brambilla, Kinetic Theory of Plasma Waves (Clarendon Press, Oxford, 1998).

[41] D. Farina et al., Nuclear Fusion 52, 033005 (2012).

[42] D. Farina and R. Pozzoli, Physics of Fluids B 2, 574 (1990).

[43] N. J. Fisch and A. H. Boozer, Physical Review Letters 45, 720 (1980).

[44] C. F. F. Karney and N. J. Fisch, Nuclear Fusion 21, 1549 (1981).

[45] E. Westerhof, Plasma Physics and Controlled Fusion 39, 1015 (1997).

[46] O. Maj, G. V. Pereverzev, and E. Poli, Physics of Plasmas 16, 062105 (2009).

[47] O. Maj, A. A. Balakin, and E. Poli, Plasma Physics and Controlled Fusion 52, 085006 (2010).

[48] R. W. Harvey, M. G. McCoy, and G. D. Kerbel, Physical Review Letters 62, 426 (1989).

[49] S. V. Kasilov and W. Kernbichler, Physics of Plasmas 3, 4115 (1996). 